\title{
EVALUATING INFORMATION SECURITY AWARENESS AND COMPLIANCE IN SUB-SAHARAN AFRICA: AN INTERPRETIVIST PERSPECTIVE
}

\author{
Sadiq Nasir and Narasimha Rao Vajjhala \\ American University of Nigeria \\ Yola, Nigeria
}

\begin{abstract}
Information security awareness and compliance are at low levels in Sub-Saharan African countries. The low level of awareness is partly because of several factors, including low Internet penetration rates, lack of adequate technological infrastructure, low levels of literacy, and high costs of acquiring new technology. However, there are other issues, including behavioral, social, cultural, and organizational issues that are often ignored. Most of the current literature on information security awareness and compliance is centered in developed countries and, to some extent, developing countries in Asia and the Middle East. There is limited literature on information security awareness and compliance, especially in Small and Medium-Sized Enterprises (SMEs) in Africa, and in particular in Sub-Saharan African countries. The purpose of this interpretivist study is to identify the significant challenges that SMEs in Nigeria, the most populous Sub-Saharan African country face concerning information security awareness and compliance. The purpose of this qualitative, multisite, exploratory case study will be to identify and explore the various social, cultural, organizational, and behavioral factors that influence the adoption and compliance with the information security policies in Nigerian SMEs. Results of this study could assist organizational leaders with maintaining higher levels of information security in their organizations and avoiding loss of brand equity and revenue because of information security breaches. Observations from audio recorded, in-depth personal interviews with 50 managers working in different departments in 20 different medium-sized companies in Nigeria will be used in this case study. The 20 medium-sized companies included two companies each from the ten key economic sectors forming the core of Nigerian economy.
\end{abstract}

\section{KEYWORDS}

Information Security, Awareness, Africa, Interpretivist, Compliance

\section{INTRODUCTION}

Technological innovations in the last decade have increased significantly bring several new challenges and opportunities. SMEs are critical for the development of developing countries (Vajjhala \& Vucetic, 2013), especially countries in Sub-Saharan Africa, such as Nigeria, because of the role played by these companies on employment generation, technological innovation, and sustainable development (Vajjhala \& Vucetic, 2013). SMEs also represent a significant portion of the national GDP and employ the most workers, especially in Sub-Saharan African countries (Abubakar, 2016), including Nigeria. One of the main challenges facing companies, especially, SMEs is information security awareness and compliance. Cyber threats at national and international levels have forced large companies as well as SMEs to examine and evaluate their current information security awareness and readiness in the context of compliance with national and international standards. Most of the existing research on information security awareness and compliance is focused on developed countries, as well as developing countries in Asia and Africa. The current studies have identified several factors, including low Internet penetration rates, lack of adequate technological infrastructure, low levels of literacy, and high costs of acquiring new technology. There is limited literature on information security awareness and compliance, especially in SMEs in Sub-Saharan African countries. Also, most of the current research on information security is focused on the technical aspects, including the hardware and software issues, with limited literature focusing on adoption and awareness. There is a growing need for studies that are carried out in these various Sub-Saharan African countries by indigenous researchers (Abubakar, 2016). As 
the term Sub-Saharan Africa is used to representing a vast geographical region, including several countries and areas under the Saharan belt, this study focuses mainly on SMEs in Nigeria, the most populous country in Sub-Saharan Africa. The purpose of this interpretivist study is to identify the significant challenges that SMEs in Sub-Saharan African countries face concerning information security awareness and compliance.

\section{LITERATURE REVIEW}

Information security as a phrase can be traced back to 1980s, and only in early-2000 did the technical professionals in the domain of information security started to identify the term. Information security as a domain of research study is still an emerging field of study (Korovessis, Furnell, Papadaki, \& Haskell-Dowland, 2017). Information security compliance assures protection of vital information systems assets. The challenge of regulating the complex domain is met with a lack of diversity in the theory and the methods used to understand this domain (Chatterjee, Sarker, \& Valacich, 2015). The severity of information security threats and the importance of information security awareness and compliance in organizations has significantly increased over the last decade (Haeussinger \& Kranz, 2017; Maynard, Tan, Ahmad, \& Ruighaver, 2018; Safa et al., 2015; Willison, Lowry, \& Paternoster, 2018). The human aspect of organizational resources is considered as the weakest link in the information security domain (Chatterjee et al., 2015). Information security awareness and compliance can help organizations address the issue around information and cybersecurity threats (Moody, Siponen, \& Pahnila, 2018).

A large number of organizations face challenges in assuring compliance with information security policies (Gaia et al., 2020). User non-compliance with security policies was identified as a significant barrier in organizational information assurance (Feng, Zhu, Wang, \& Liang, 2019). Over half of the security breaches are caused by a low level of compliance by the system users (Feng et al., 2019). There is a need for understanding the factors that can help organizations in assuring higher levels of information security awareness and compliance. Countries in Sub-Saharan Africa are characterized by different social, cultural, and economic dimensions as compared to countries in the Americas, Asia, and Europe. Hence, even though findings from studies on information security compliance carried out in other countries may help understand some of the challenges, there is a definite need for studies to be carried out in the Sub-Saharan African countries to help understand and validate these findings as well as explore any additional factors.

Bulgurcu, Cavusoglu, and Benbasat (2010) suggested that the attitude of an employee determines their level of compliance with information security policies. Bulgurcu et al. (2010) suggested that the theory of planned behavior underpins the attitude of the employees and also identified a direct connection between the attitude of the employees and the cost of compliance, the benefit accrued from compliance, cost of non-compliance, and belief over general compliance and non-compliance. Haeussinger and Kranz (2017) define security awareness as a state of knowledge and consciousness about issues around security. Hence, information security awareness should self-promote security compliance behavior. There is a need to fill in the knowledge void by examining the factors that influence compliance with information security policies. Haeussinger and Kranz (2017) established a connection between information security awareness and behavioral intention.

Training and educating employees is essential to achieve higher levels of compliance with information security. Eminağaoğlu, Uçar, and Eren (2009) proposed that the success of compliance of personnel is attained through multiple means, mainly training and education. However, several researchers have suggested that poorly structured and planned information security training programs can adversely influence the effectiveness of training and education programs. Some of the studies have indicated that most of these information security awareness programs lacked a theoretical grounding and empirical evidence of practical applicability (Kirova \& Baumöl, 2018).

\section{RESEARCH APPROACH}

The interpretivist paradigm in research provides the theoretical lens that would provide the direction for the research. The use of positivism in this research is not ideal because positivism uses hypotheses, while an interpretivist approach would use propositions. The assumption of positivism limits its exploration potential, and exploring the social context in which a phenomenon is occurring is not possible. Hence, in this study, an 
interpretivist approach would be adopted. The purpose of adopting the interpretivist approach is to obtain meaning from the social process by which societal constructs are formulated. The adoption of an inductive approach as a strategy will allow the research to gather knowledge from experience. The interpretivist approach enables the researchers to start by interfacing with observations and theories to drill down for answers. This approach will also allow the researcher to conduct observations and to come up with an explanation for the research phenomenon.

In this qualitative, multisite, exploratory case study the influence of various social, cultural, organizational, and behavioral factors on compliance with information security policies in medium-sized enterprises in Nigeria will be examined. Case studies provide an opportunity to combine several data collection techniques, including interviews, questionnaires, archived documents, and observations (Vajjhala \& Baghurst, 2014). The study is driven by the central research question: How do various social, organizational, cultural, and behavioral factors influence employees' perceptions about compliance with information security policies? Qualitative in-depth interviews will be conducted with 50 managers working in 20 medium-sized enterprises in Nigeria. Open-ended interview questions will be used as the data collection instrument for this qualitative, multisite, exploratory case study. The interview questions will be formulated in a manner to obtain descriptive data from the participants that would address the central research question. The criteria set forth by the African Union and Nigerian governments would be considered and used for establishing whether a firm was an SME. The qualitative data collected in this study will be analyzed using qualitative techniques, including assigning codes to the phrases, and then analyzing the keywords in context using visual pattern comparisons to identify and group similar words together with the help of NVivo or similar content analysis software.

\section{CONCLUSION}

The findings from this study could assist organizational leaders with maintaining higher levels of information security in their organizations and avoiding loss of brand equity and revenue because of information security breaches. The findings from this study should also add value to the existing literature on the factors contributing to higher levels of information security compliance in Nigerian SMEs as well as other countries in the Sub-Saharan African region. The limitations of this study are generalizability and applicability of the findings to other countries in the Sub-Saharan African region. The Sub-Saharan African region is quite large and has a number of countries and regions with diverse set of economic, political, and cultural differences. Hence, the findings of this study need to be replicated and validated across the region to explore the applicability as well as identify any additional factors that were not identified in this study. Future research could help address the issue of generalizability by replicating this study across the regions and demographics.

\section{REFERENCES}

Abubakar, D. A. (2016). Cloud computing adoption by SMEs in Sub-Saharan Africa. (Ph.D. Doctoral), Robert Gordon University, Aberdeen, Ireland.

Bulgurcu, B., Cavusoglu, H., \& Benbasat, I. (2010). Information security policy compliance: An empirical study of rationality-based beliefs and information security awareness. Management Information Systems Quarterly, 34(3), 523-548.

Chatterjee, S., Sarker, S., \& Valacich, J. S. (2015). The behavioral roots of information systems security: Exploring key factors related to unethical IT use. Journal of Management Information Systems, 31(4), 49-87.

Eminağaoğlu, M., Uçar, E., \& Eren, Ş. (2009). The positive outcomes of information security awareness training in companies - A case study. Information Security Techology Report, 14(4), 195-201.

Feng, G., Zhu, J., Wang, N., \& Liang, H. (2019). How Paternalistic Leadership Influences IT Security Policy Compliance: The Mediating Role of the Social Bond. Journal of the Association for Information Systems, 20(11), 1650-1691.

Gaia, J., Ramamurthy, B., Sanders, G., Sanders, S., Upadhyaya, S., Wang, X., \& Yoo, C. (2020). Psychological profiling of hacking potential. Paper presented at the Hawaii International Conference on System Sciences, Hawaii, USA.

Haeussinger, F., \& Kranz, J. (2017). Antecedents of employees' information security awareness -Review, synthesis, and directions for future research. Paper presented at the European Conference on Information Systems, Guimarães, Portugal. 
Kirova, D., \& Baumöl, U. (2018). Factors affecting the success of security education, training and awareness programs - A literature review. Journal of Information Technology Theory and Application, 19(2), 24-38.

Korovessis, P., Furnell, S., Papadaki, M., \& Haskell-Dowland, P. (2017). A toolkit approach to information security awareness and education. Journal of Cybersecurity Education, Research and Practice, 2(5), 1-32.

Maynard, S. B., Tan, T., Ahmad, A., \& Ruighaver, T. (2018). Towards a framework for strategic security context in information security governance. Pacific Asia Journal of the Association for Information Systems, 10(4), 65-88.

Moody, G. D., Siponen, M., \& Pahnila, S. (2018). Toward a Unified Model of Information Security Policy Compliance. MIS Quarterly, 42(1), 285-311.

Safa, N. S., Sookhak, M., Von Solms, R., Furnell, S., Ghani, N. A., \& Herawan, T. (2015). Information security conscious care behaviour formation in organizations. Computers \& Security, 53(1), 65-78.

Vajjhala, N. R., \& Baghurst, T. (2014). Influence of cultural factors on knowledge sharing in medium-sized enterprises within transition economies. International Journal of Knowledge Management Studies, 6(4), 304-321.

Vajjhala, N. R., \& Vucetic, J. (2013). Key barriers to knowledge sharing in medium-sized enterprises in transition economies. International Journal of Business and Social Sciences, 14(4), 90-98.

Willison, R., Lowry, P. B., \& Paternoster, R. (2018). A tale of two deterrents: Considering the role of absolute and restrictive deterrence to inspire new directions in behavioral and organizational security research. Journal of the Association for Information Systems, 19(12), 1187-1216. 